\title{
Automobile Censoring and Chasing Method for Avoiding Accidents to Intensify Safety and Security
}

\author{
${ }^{1}$ Ramya D, ${ }^{2}$ Santhiya Grace A, ${ }^{3}$ Devi Kala Rathinam, ${ }^{4}$ Sherin J, ${ }^{5}$ Shilpa A, ${ }^{6}$ Rajkumar Y \\ ${ }^{1}$ Department of Computer Science \& Engineering, Sri Krishna College of Engineering \& Technology, Coimbatore. Tamilnadu, India. \\ ${ }^{2}$ Department of Computer Science \& Engineering, Sri Krishna College of Engineering \& Technology, Coimbatore. Tamilnadu, India. \\ ${ }^{3}$ Department of Computer Science \& Engineering, Sri Krishna College of Engineering \& Technology, Coimbatore. Tamilnadu, India. \\ ${ }^{4}$ Department of Computer Science \& Engineering, Sri Krishna College of Engineering \& Technology, Coimbatore. Tamilnadu, India. \\ ${ }^{5}$ Department of Computer Science \& Engineering, Sri Krishna College of Engineering \& Technology, Coimbatore. Tamilnadu, India. \\ ${ }^{6}$ Department of Computer Science \& Engineering, Sri Krishna College of Engineering \& Technology, Coimbatore. Tamilnadu, India.
}

Correspondence Author: Ramya D, Department of Computer Science \& Engineering, Sri Krishna College of Engineering \& Technology, Coimbatore. Tamilnadu, India.

E-mail: ramya@skcet.ac.in

Received date: 21 February 2018, Accepted date: 26 April 2018, Online date: 28 April 2018

Copyright: (C) 2018 Ramya D, et al. This is an open-access article distributed under the terms of the Creative Commons Attribution License, which permits unrestricted use, distribution, and reproduction in any medium, provided the original author and source are credited.

\begin{abstract}
Though advancements in technology progressed to a greater extent, it is really difficult to control the vehicular accidents and hazards especially in the metropolitan cities. Due to the malign construction of roads and poor quality design of roads vehicles the accidents are more prevalent. To face these circumstances an innovative vehicle censoring and chasing ideology has been proposed and structured for the ambition of censoring the automobiles which are gripping from one location to another to give safety and security. The proposed methodology encompasses existing technology by means of Embedded C Programming language and ARM7TDMI-S unit is developed for the practical appearance of loading data in the database. It encompasses GPS and GSM for vehicular censoring and chasing including SIM800 which acts like a mobile phone with its unique phone number. It can transmit voice, SMS, and data information with low power consumption. The GPS gives current location of the automobile, GPRS sends the current chasing data to the server and so an active SMS gets generated and sent to the riders of the automobile. This method is implemented in the interior of the vehicle whose area is to be resolved on the web page and administered at actual time. If the owner or the driver drives in bad course like drowsiness or drunken, the processor caution by producing a sound through alarming sensor that produces a beep sound. The proposed method completely looks after the driver's care by avoiding from the hazards.
\end{abstract}

Key words: Alarm, Censoring, Chasing, GPS (Global Positioning System), GSM (Global System for Mobile Communication), Security engines, ARM7TDMI-S (32 bit microprocessor), SIM800 (Quad Band GSM/GPRS Solution), GPRS (General Packet Radio Service).

\section{INTRODUCTION}

GSM and GPS based Vehicle Location and Tracking System proposed by Babrurao et.al [1] used not only for tracking the vehicle but also for positioning the vehicle. Embedded system is used in this system. In this embedded system microcontroller AT89S52 is used for interfacing with hardware devices. The microcontroller AT89S52 used as an interface for both GPS and GSM. This setup used to monitor the movement of vehicles continuously. The GPS modem sends the vehicle position continuously in the form of Latitude and Longitude. GSM is same as GPS, but it sends the vehicle position from remote area in the form of latitude and longitude. Among many outputs of GPS, NMEA data is displayed in LCD. The mobile numbers are stored in EEPROM, using that EEPROM the data's are send to mobile phones. The serial communication between modems and microcontroller is done using RS-232 protocol. The TTL voltage is converted to RS-232 using IC serial driver. If the user needs the location of the vehicle they send the request to the modem. Automatically the vehicle latitude and longitude are sending as reply. Using the latitude and longitude the vehicle position can be found. This method is used mainly to find the stolen vehicles. This system is not more secured because any of the users can send the request to know about the location of the vehicle. Without any verification process the system will send the latitude and longitude of vehicle to user. Sometimes this reply may produce some problems. In future this system can be upgraded by locking the vehicle with single SMS. Using this locking technique, the vehicle can be protected from thief. The emergency numbers can be added in the system for emergency purpose. Up gradation of the system is very simple and easy process.

Design and Implementation of Vehicle Tracking System Using GPS proposed by Ambade.et.al [2] finds the location of the vehicle. This system helps the owner to know the vehicles current location and product the system from thief. Some sensors like proximity sensor and ultrasonic sensors are used in this system. Proximity sensors are used for parking the vehicle. Ultrasonic sensors are used to measure the distance of the vehicle. In this system database is created to store all the data's produced by the vehicle. The server is used to store the information which is known as tracking server. In vehicle unit is the software which is installed in the vehicle and also in the administrator system that monitor the movement of vehicle. Many vehicles can have the same system. The information of one system should not collapse with the information of other system. To avoid this database is maintained to store the information.

The following are the data stored in the database as follows:

$>$ Information about vehicle tracking system user

$>\quad$ Information about the vehicle in which the vehicle tracking system is fixed 


\section{$>$ All other Information received from vehicle}

In this system GPS is used to find the current location of the vehicle. In this system microcontroller ARM9 is used. It used to monitor and control the functions of In-Vehicle unit. The input is given to the microcontroller using the flash memory. The microcontroller read the instruction one by one and the operation can be performed.

The Design and Implementation of Vehicle Tracking system Using GPS/GSM GPRS Technology and Smartphone Application [3] proposed by SeokJu et.al. designed an effective automobile or the vehicle chasing system is described and actualized for chasing the action of each and every armed automobile from any area at any moment. The advanced method had better usage of a prominent automation which includes a Smartphone along with a microcontroller (MCU). It is very cheap when compared with others. The constructed automobile equipment works with the help of Global Positioning System (GPS) and Global System for Mobile Communication, General Packet Radio Service (GSM/GPRS) mechanism which is the important method for automobile chasing. The equipment is fixed under a vehicle for which location is to be described and chased in actual. A microcontroller is accessed to curb the GPS and GSM/GPRS sections. The automobile chasing method aids the GPS section to access environmental locations at balanced period of time. The GSM/GPRS section broadcasts and restores the vehicle area to the directory. A Smartphone device is also established for repeatedly notifying the vehicle area. API is owned to exhibit the automobile on the map in the Smartphone. Hence, end users is going to be repeatedly sense a motion vehicle as required with the help of Smartphone and examine the approximated interval and point for the vehicle to reach the particular area. With the aim of indicating the possibility and performance of the arrangement, this paper gives the automobile chasing method with practical executions.

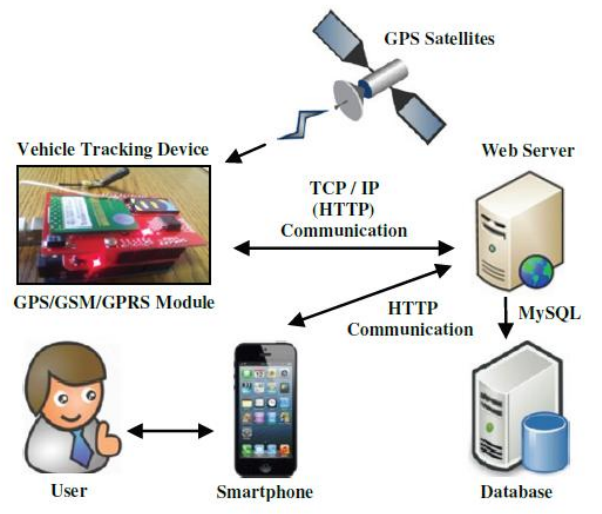

Fig. 1: Vehicle Tracking System

The Hybrid GPS-GSM Localization of Automobile Tracking System [4] proposed by Mohammad A. Al-Khedher et.al incorporated GPS-GSM method is introduced to chase automobile's area and its category is the main aim of convertible course nursing organization. These organizations are carried out with the help of various fusion methods which brings in mobile consultation, environmental disposing and encapsulated methods. This vehicle chasing methods are constructed to aid associations with huge number of vehicles and various custom goal. The acknowledged GPS locations are drained with the help of the Kalman filter to develop the efficiency of calculated area. Apart from this obligation may be anticipated in prior stood on present vehicle area. The GPS is asteroid/spacecraftbased exploration method. At the areas close to the earth, the indications are emulated by towering. Various factors including the area identification, has clock error in the spacecraft timer. To rectify this error few GPS towers, evaluate the relation of the information from one tower to another tower. Kalman filter can also be extended for frontier detection system in the sea where the fishermen problem can be mitigated in the Indian ocean [5].

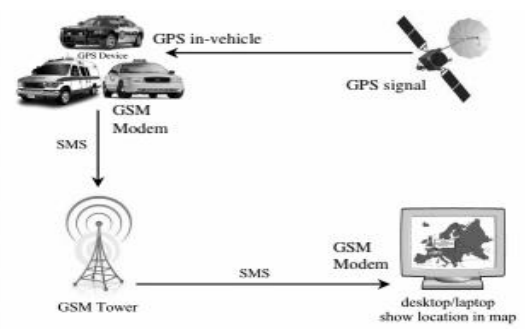

Fig. 2: Vehicle Censoring System

Working Principle:

Alcohol Sensor:

Alcohol sensor fitted near the driver seat, so it can sense the alcohol content in the breath of the driver. If the driver as consumed alcohol and it has been detected by the sensor automatically the lock gets jammed, so the driver can't even able to start his vehicle. It is a low-cost sensor. An alcohol sensor detects the attentiveness of alcohol gas in the air and an analog voltage is an output reading. The MQ-135 alcohol sensor consists of a tin dioxide (SnO2), a perspective layer inside aluminum oxide micro tubes (measuring electrodes) and a heating element inside a tubular casing. The end face of the sensor is enclosed by a stainless-steel net and the back side holds the connection terminals. Ethyl alcohol present in the breath is oxidized into acetic acid passing through the heat element. With the ethyl alcohol cascade on the tin dioxide sensing layer, the resistance decreases. By using the external load resistance, the resistance variation is converted into a suitable voltage variation. The circuit diagram is shown below 


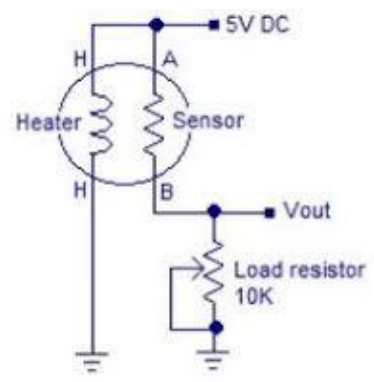

Fig. 3: MQ-Circuit diagram

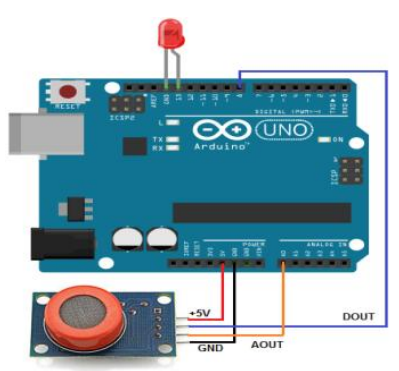

Fig. 4: Alcohol Sensor

\section{Wheel Sensor:}

The traditional wheel speed sensor consists of a tone wheel. Inside the sensor itself there is a permanent magnet surrounded by a coil of copper wire. The magnetic field changes as the teeth of the tone wheel pass, creating an alternating voltage (AC) signal the changes in both frequency and strength based on speed. It is a typical sine wave. The sensor has two wires: ground and signal. This type of wheel speed sensor is called a passive sensor because there is no power going over it. A magnetic wheel speed sensor does not start its working until the vehicle is travelling about $5 \mathrm{mph}$ or faster. The tone wheel is mounted on the axle, the brake rotor, the drum or the constant velocity joint. The gap between the two is critic and, although some sensors may be adjusted. Small chip in the wheel may cause problems during working. So, use of tools like hammer, pry bar causes certain irregularities inside it. This causes the sensors to show incorrect signals. The ABS module needs both signal amplitude and frequency. Even small differences can affect the ABS module. The sensor will be active only if power supply is connected to it. The active sensor is a proximity sensor with integrated electronics supplied with a voltage. The voltage is supplied by the ABS unit. For the pulse wheel, a multipolar ring may be used and positioned in a sealing ring of the wheel bearing. Magnets with alternating poles are installed in the sealing ring the magneto-resistors integrated in the sensor electronics detect a rotating magnetic field when the multipolar ring rotates. The electronics in the sensor convert the resulting sinusoidal signal into a digital signal. The signal is transmitted to the control unit in the form of a current signal using pulse width modulation. The sensor is connected to the control unit via a two-wire electric cable. The sensor signal is transmitted via the power supply wire.

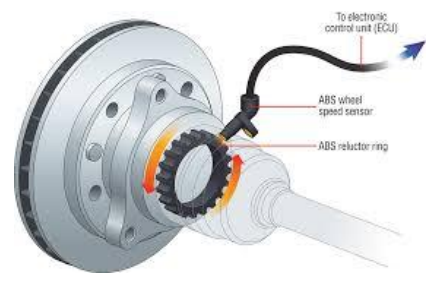

Fig. 5: Wheel sensor

\section{Proposed System:}

Sensor based accident detection and avoiding technique plays a major role in the present world. An efficient technique to overcome the drawbacks of the existing system where most of the accidents scouring today are because of drunk and drive and in adequate speed control measures. So, in order to overcast these problems this technique is used. The proposed system based on sensors to prevent accidents. Various sensors are used to monitor the speed and bring efficient safety measures to avoid accidents. Along with prevention technique we propose a new system using wheel sensor and alcohol sensor to prevent the accident occurrence to the nearby rescue. This alcohol sensor is suitable for detecting alcohol concentration on your breath, like the common breathalyzer It has a high sensitivity and fast response time. Sensor provides an analog resistive output based on alcohol concentration. The drive circuit is very simple all it needs is one resist. Wheel sensors are used to control speed, more features of wheel sensor are anti-lock brake system, traction control and electronically stability control. Two types of wheel speed sensors are available they are the magnetic sensors and the Hall Effect sensors which are more popular in the past few years. The proposed system when incorporated with Internet of Things provides continuous tracking inspite of practical hurdles in networks too [5].

\section{REFERENCES}

[1] Kodavati, Baburao, V.K. Raju, S. Srinivasa Rao, A.V. Prabu, T. Appa Rao, Dr.Y.V. Narayana, 2011. "GSM and GPS based vehicle location and tracking system. " International Journal of Engineering Research and Applications (IJERA) ISSN :2248-9622.

[2] Ambade Shruti Dinkar and S.A Shaikh, 2011. "Design and implementation of vehicle tracking system using GPS." Journal of Information Engineering and Applications www.iiste.org ISSN 2224-5758 (print) ISSN 2224-896X (online) 1(3): 1-6.

[3] Lee, SeokJu, Girma Tewolde, and Jaerock Kwon, 2014.", Design and implementation of vehicle tracking system using GPS/GSM/GPRS technology and smartphone application."Internet of Things (WF-IoT), 2014 IEEE World Forum on. IEEE.

[4] Mohammad A. Al-Khedher, 2012. "Hybrid GPS-GSM localization of automobile tracking system." International Journal of Computer Science \& Information Technology (IJCSIT) 3(6) 2011.

[5] D.Ramya, V.C.Mahavishnu, Y.Rajkumar, K.Sujatha, S. Mohana Gowri, R.Vidya, 2017 . "IoT based Frontier Security and Tracking System for Indian Fishermen using a Threshold based Algorithm”, International Journal of Pure and Applied Mathematics, Volume 117 No. 22, 271-274. 